\title{
Prognostic values of cardiac biomarkers screening in dialysis patients
}

\author{
Simona Hogas ${ }^{\star, 1}$, Petru Cianga ${ }^{2}$, Daniela Constantinescu², Corina Cianga ${ }^{2}$, Luminita \\ Voroneanu ${ }^{1}$, Raluca Popa ${ }^{1}$, Stefana Catalina Bîlha ${ }^{3}$, Adrian Covic ${ }^{1}$
}

${ }^{1}$ Nephrology Department, "Dr. C.I. Parhon" University Hospital, "Grigore T. Popa" University of Medicine and Pharmacy, Iași, Romania, ${ }^{2}$ Department of Immunology, "Grigore T. Popa" University of Medicine and Pharmacy, lași, Romania, ${ }^{3}$ Endocrinology Department,"Sf. Spiridon" Hospital, "Grigore T. Popa" University of Medicine and Pharmacy, lasi, Romania

\begin{abstract}
Patients with chronic kidney disease (CKD) have an increased cardiovascular (CV) risk and a higher mortality rate. This case report aims to reveal the prognostic value of cardiac biomarkers in patients with CKD stage 5 on dialysis. Several biomarkers have proven their utility for early detection of CV risk in dialysis patients. Most promising biomarkers are: N-terminal pro-B-type (NT-proBNP), high sensitivity cardiac troponin T (HscTnT), Cardiotrophin-1 (CT-1) and Galectin-3 (GAL-3). We report the case of a 44 year-old woman with end stage renal disease on dialysis without any cardiac pathology, but who had, at screening evaluation, high values of cardiac biomarkers, with increasing levels of NT-proBNP at serial determination; Hs-cTnT was constant. Recently, our patient was admitted in cardiology unit with unstable ischemic cardiopathy. In this context, we review the prognostic value of cardiac biomarkers in CV morbidity and mortality. The particularity of this case was the preemptive assessment of cardiac biomarkers. High serum levels of these biomarkers, in a patient without any cardiac concerns at evaluation moment, should promote for early invasive investigations.
\end{abstract}

Keywords: chronic kidney disease, dialysis, high sensitivity cardiac troponin $T, N$-terminal pro-B-type, cardiotrophin-1, galectin -3

\section{Introduction}

Patients with chronic kidney disease have an increased mortality risk; CV events are the leading cause of mortality in these patients. This risk is even higher in dialysis patients. Numerous interventional clinical trials have addressed overall and CV mortality in CKD; however, almost all were negative [1].

Underpowered studies, selection of

Received: August 2015; Accepted after review: September 2015; Published: September 2015.

*Corresponding author: Simona Hogas, MD, Nephrology Department, "Dr. C.I. Parhon" University Hospital, "Grigore T. Popa" University of Medicine and Pharmacy, 16 Universitatii str, Iași, Romania.

E-mail: mmh1228@yahoo.com 'healthier' patients, better quality of care during trial, competing risks for mortality and mostly late intervention are possible explanations.

Therefore, early evaluation of $\mathrm{CV}$ risk in this population is paramount, serving to reduce morbidity and mortality in this population. Several biomarkers might be of value for early detection of cardiovascular risk. Among them, cardiac biomarkers could be useful to assess $\mathrm{CV}$ risk in dialysis patients.

$\mathrm{N}$-terminal pro-B-type is primarily used to detect, diagnose, and evaluate the severity of heart failure. It can be used, along with other cardiac biomarker tests, to detect heart failure. Previous studies have shown that NT-proBNP is a strong predictor of overall mortality in asymptomatic hemodialysis patients [2]. High sensitivity cardiac troponin $\mathrm{T}$ is a marker for 
prediction cardiovascular events, in general increased even in the absence of acute coronary syndrome in dialysis patients. However, several studies reveal that serum level of Hs-cTnT may be a marker of survival in hemodialysis patients [2, 4]. Cardiotrophin-1 is a new a member of the interleukin 6 family, a cytokine that promotes cardiac fibrosis, structural cardiac changes, by increasing collagen synthesis and myocyte hypertrophy that leads to left ventricular dysfunction [5]. CT-1 also favors the development of atherosclerotic plaques, promoting vascular calcification [6]. Serum levels of CT-1 are increased in patients with heart failure, hypertensive heart disease [7, 8].

Galectin-3 is a member of the lectin family, expressed predominantly by macrophages. GAL-3 is involved in many immunity processes and also promotes cell growth and differentiation, apoptosis and angiogenesis causing cardiovascular hypertrophy and fibrosis. GAL-3 is an important mediator that induces fibroblasts to proliferate and to increase collagen synthesis [9].

Several studies revealed that serum level of GAL-3 is related with mortality. The PREVEND study showed that patients with median level of $5.6 \mathrm{ng} / \mathrm{mL}$ had a $15 \%$ 10-year mortality rate compared with a $5 \%$ rate for those with median $7.7 \mathrm{ng} / \mathrm{mL}$ [10]. In cohort from Framingham study, GAL-3 was associated with increased left ventricular mass and with all cause mortality [11]. Also in the PROVE IT-TIMI 22 study, 100 patients who developed heart failure after acute coronary syndrome had a higher baseline GAL-3 levels median $16.7 \mathrm{ng} / \mathrm{L}$ vs $14.6 \mathrm{ng} / \mathrm{L}$ [12].

\section{Case report}

A 44 year-old woman was admitted to hospital because of sudden chest pain and shortness of breath. Eight years ago, she was diagnosed with CKD, directly in uremic stage, without identifiable etiology. Initially, she performed peritoneal dialysis for 5 years, then, due to recurrent episodes of peritonitis associated with peritoneal dialysis, she was converted to hemodialysis. Six years before the current admission the patient was population [3]. Serum levels of troponin can be diagnosed with papillary carcinoma of the thyroid (PTC) followed by thyroidectomy. PTC is generally a slow growing tumor with favorable prognosis. Our patient also had a good therapeutic response, without any relapses.

On examination, the blood pressure was 160/70 $\mathrm{mmHg}$, heart rate 75 beats per minute. The other vital signs and oxygen saturation were normal. An ECG showed sinus rhythm at a rate of 75 beats per minute, with left ventricular hypertrophy (LVH), without any ischemic characteristic changes. Cardiac ultrasonography revealed LVH and decreased ventricular contractile function (ejection fraction 35\%). The hematocrit, hemoglobin level, white-cell count, platelet count, and erythrocyte sedimentation rate were normal, as were blood levels of electrolytes, calcium, phosphorus, magnesium, glucose. Cardiac enzymes were also within normal limits. Coronary angiography evaluation revealed $90 \%$ occlusion of left anterior descending artery, which required stenting. After the procedure the patient's condition has improved.

Three years before the current presentation, we collected blood samples and serum was stored at minus 80 Celsius degrees. We used the samples to determine serum levels of cardiac biomarkers, as screening. The biomarkers we tested were cardiotrophin-1, galectin-3, NT-proBNP and high sensitivity $T$ troponin. We determined serum level of CT-1 and GAL-3 only from the sample collected three years before the actual event. For NT-proBNP and Hs-cTnT we performed 3 consecutive determinations, one test per year. Results of serum parameters are presented in Table 1.

We noted not only a high basal value, but also a progressive increase in serum levels of NT pro-BNP from the first determination, to the third determination performed one year before current event. Serum levels of NT-proBNP regarded as having unfavorable cardiovascular prognosis if it exceeds $300 \mathrm{pg} /$ $\mathrm{mL}$ [13]. The levels of Hs-cTnT were in normal limits in all determinations. The upper reference limit of Hs-cTnT in general population is $14 \mathrm{ng} / \mathrm{L}$ [3]. Galectin-3 level was 
comparable to the values described before in dialysis patients [14]. Also CT-1 serum level dosed from the samples stored three years ago was far higher than the higher data reported in the literature. Currently there are no data for normal values of CT-1 in dialysis patients but the cut-off value for CT-1 plasma levels of $152 \mathrm{pg} / \mathrm{mL}$ has a higher sensitivity and specificity for detecting the incidence of heart failure in hypertensive patients [15].

Table 1. Cardiac biomarkers evaluation

\begin{tabular}{lccc}
\hline \multicolumn{2}{l}{ 3 years before event } & 2 years before event & 1 year before event \\
\hline NT pro-BNP $(\mathrm{pg} / \mathrm{ml})$ & & & \\
\hline Hs-cTnT $(\mathrm{pg} / \mathrm{ml})$ & 2730 & 4567 & 5033 \\
CT-1 $(\mathrm{pg} / \mathrm{ml})$ & 11.21 & 14.35 & 12.41 \\
GAL-3 $(\mathrm{ng} / \mathrm{ml})$ & 2000 & & \\
\hline
\end{tabular}

\section{Discussions}

Elevated levels of natriuretic peptides are associated with poor long term prognosis and have an adverse impact on long-term mortality in patients with congestive heart failure [14] and coronary artery disease $[15,16]$.

The current American College of Cardiology/American Heart Association (ACC/AHA) guidelines for managing heart failure have incorporated using natriuretic peptide levels in establishing the prognosis and disease severity of chronic heart failure and acutely decompensated heart failure [17]. Natriuretic peptides levels are also correlated with cardiovascular events and cardiovascular mortality both the general population and in patients with CKD. Because cardiac troponins are usually increased in dialysis patients, even without acute coronary syndrome, the prognostic value of this biomarker is not yet fully elucidated [18].

Previously, our team performed a study which assessed the role of hs-cTnT alone or in combination with NT-proBNP in predicting death and fatal or non-fatal major CV events. The study showed that NT-proBNP was a strong predictor of overall mortality in asymptomatic hemodialysis patients. The addition of hs-cTnT did not improve the prognostic accuracy compared with NTproBNP alone [2].

CT-1 is a promoter of atherosclerosis by stimulating collagen synthesis and promoting cardiac fibrosis [19]. Experimental studies show that CT-1 may also cause vascular dysfunction, by favoring the development of vascular plaques [20]. This would explain CT-1 role as prognostic factors for cardiovascular events. In our patient the serum level of CT-1 was increased 1 year before the event. So it could assess patient angiography preemptively to prevent acute cardiovascular event.

Recently FDA approved the use of galectin-3 for prognosis of chronic heart failure [9]. The 2013 American College of Cardiology Foundation/American Heart Association (ACCF/AHA) Guideline for management of heart failure also states that galectin- 3 may be considered an additional biomarker for both chronic and acute heart failure improved risk stratification [17]. In this case, the serum level of GAL-3 was higher 3 years before the current event.

To the best of our knowledge, this case is unique, because report the prognostic values of preemptive and simultaneous evaluation of multiple biomarkers for evaluation the cardiovascular events in dialysis patients.

\section{Conclusions}

The current case reveals that biomarkers like NT-proBNP, CT-1 and GAL-3, may have prognostic value for early detection of patients at risk for cardiovascular events.

Track the category in particular patients, with CKD stage 5 on dialysis assessment and early detection of cardiovascular risk can adapt therapeutic strategies and may reduce morbidity and mortality in this population. 


\section{Acknowledgement}

This paper was published under the frame of European Social Fund, Human Resources Development Operational Program 2007-2013, project no.: POSDRU/159/1.5/S/135760, CERO - Career Profile: Romanian Researcher.

\section{References}

1. Collins AJ, Foley RN, Chavers B, et al. United States renal data system 2011 annual data report: atlas of chronic kidney disease \& endstage renal disease in the United States. $A m J$ Kidney Dis 2012; 59:A7; e1-420.

2. Voroneanu L, Siriopol D, Nistor I, et al. Superior predictive value for NTproBNP compared with high sensitivity cTnT in dialysis patients: a pilot prospective observational study. Kidney Blood Press Res 2014; 39(6):636-647.

3. Reichlin $\mathrm{T}$, Hochholzer W, Bassetti $\mathrm{S}$, et al. Early diagnosis of myocardial infarction with sensitive cardiac troponin assays. $N$ Engl $J$ Med 2009; 361:858-867.

4. Artunc F, Mueller $\mathrm{C}$, Breidthardt $\mathrm{T}$, et al. Sensitive troponins--which suits better for hemodialysis patients? Associated factors and prediction of mortality. PLOS One 2012; 7:e47610.

5. Pennica D, King KL, Shaw KJ et al. Expression cloning of cardiotrophin 1 a cytokine that induces cardiac myocyte hypertrophy. Proc. Natl. Acad. Sci 1995; 92:1142-1146.

6. Lopez B, Gonzalez A, Querejeta R, Larman M, Rabago G, Diez J. Association of cardiotrophin1 with myocardial fibrosis in hypertensive patients with heart failure. Hypertension 2014; 63:483-489.

7. López B, González A, Lasarte JJ, et al. Is plasma cardiotrophin-1 a marker of hypertensive heart disease? J Hypertens 2005; 23:625-632.

8. Filipe MD, Meijers WC, van der Velde AR, Boer RA. Galectin-3 and heart failure: prognosis, prediction and clinical utility. Clin Chim Acta 2015; 443:48-56.

9. de Boer A, van Veldhuisen DJ, Gansevoort RT, et al. The fibrosis marker galectin-3 and outcome in the general population. $J$ Intern Med 2012; 272:55-64.

10. Ho JE, Liu C, Lyass A, et al. Galectin-3, a marker of cardiac fibrosis, predicts incident heart failure in the community. J Am Coll Cardiol 2012; 60:1249-1256.

11. Grandin EW, Jarolim $P$, Murphy $S A$, et al. Galectin-3 and the development of heart failure after acute coronary syndrome: pilot experience from PROVE IT-TIMI 22. Clin Chem 2012; 58:267-273.

12. Kwan G, Isakson SR, Beede J, Clopton $P$, Maisel AS, Fitzgerald RL. Short-term serial sampling of natriuretic peptides in patients presenting with chest pain. J Am Coll Cardiol 2007; 49(11):1186-1192.

13. Christiane Drechsler, Graciela Delgado, Christoph Wanner, et al. Galectin-3, Renal Function, and Clinical Outcomes: Results from the LURIC and 4D Studies. JASN 2015. ASN.2014010093; J Am Soc Nephrol 2015; 7; 26(9):2213-2221.

14. Bielecka-Dabrowa $A$, Gluba-Brzozka $A$, Michalska-Kasiczak M, Misztal M, Rysz J, Banach M. The multi-biomarker approach for heart failure in patients with hypertension. Int $J$ Mol Sci 2015; 16:10715-10733.

15. Berger R, Huelsman M, Strecker $K$, et al. Btype natriuretic peptide predicts sudden death in patients with chronic heart failure. Circulation 2002; 105(20):2392-7.

16. Schnabel R, Lubos E, Rupprecht HJ, et al. Btype natriuretic peptide and the risk of cardiovascular events and death in patients with stable angina: results from the AtheroGene study. J Am Coll Cardiol 2006; 47(3):552-558.

17. Kragelund $C$, Gronning $B$, Kober $L$, Hildebrandt P, Steffensen R. N-terminal pro-B-type natriuretic peptide and long-term mortality in stable coronary heart disease. $N$ Engl J Med 2005; 352(7):666-675.

18. Yancy CW, Jessup M, Bozkurt B, et al. 2013 ACCF/AHA guideline for the management of heart failure: a report of the American College of Cardiology Foundation/American Heart Association Task Force on practice guidelines. Circulation 2013; 128(16):e240-327.

19. Paniagua R, Ventura MD, Avila-Díaz $M$, et al. NT-proBNP, fluid volume overload and dialysis modality are independent predictors of mortality in ESRD patients. Nephrol Dial Transplant 2010; 25:551-557.

20. Konii H, Sato K, Kikuchi S, et al. Stimulatory effects of cardiotrophin-1 on atherosclerosis. Hypertension 2013; 62:942-950. 
21. Lopez Andres N, Calvier L, Labat C, et al. Absence of cardiotrophin 1 is associated with decreased age-dependent arterial stiffness and increased longevity in mice. Hypertension 2013; 61:120-129. 